\title{
A Load Balancing Model for Job Scheduling using Cooperative BEE Scout
}

\author{
Kapil Dangi \\ Samrat Ashok Technology Institute \\ Vidisha (M.P)
}

\author{
Nirmal Gaud \\ Samrat Ashok Technology Institute \\ Vidisha (M.P)
}

\begin{abstract}
The efficiency and proper utilization of cloud environments depends on the balancing of load. The limited number of resource and on demand access of resource creates the situation of overloading. The process of overloading degraded the performance of cloud environments. Now days used various swarm based algorithm for load balancing. In this paper proposed coupling based load balancing model based on BEE scout. The BEE scout model coupled the virtual machine during the allocation of resource. . The scout based technique basically used the concept of sharing of virtual machine. The shared virtual machine allocated the job in dedicated time period for the execution of process. The proposed model simulated in cloudsim simulator and used various parameters such as data center, number of user base and many more. The proposed model simulate in cloudsim simulator.
\end{abstract}

\section{General Terms}

Cloud Computing, CGT, Honey Bee

\section{Keywords}

Cloud Computing, Load Balancing,

\section{INTRODUCTION}

The load balancing is important area of cloud computing environment. The process of balancing balanced the limited number of resource and unlimited number of job arrival. The process of balancing of workload improved the performance of cloud environment. The process of load balancing undergone in two different scenario static and dynamic. The static balancing technique used the process of CPU scheduling and some common data searching technique. The dynamic load balancing adds some extra process and increase the cost of balancer. Now a day the dynamic load balancing technique used heuristic based function. The heuristic based function swarm intelligence for the purpose of balancing. The dynamic load balancing model consist of distributed computing technique for the controlling of load over the cloud environment. Now a day the public cloud computing environment used cloud partition technique. the cloud partition technique, divided the cloud load into serval part and then apply the process of balancing. In this paper used the graph theory based load balancing technique, these techniques divide the load in three situations one is under load, two is ideal situation and finally situation is overload. The all situated virtual machine mapped according to their total capacity of load. The all capacity of virtual machine sharing based. The shared load by virtual machine map the situation of cloud environment. The haring of graph node imports the load of virtual machine for one node to another node. The time allocation frame slot used the concept of queuing theory for the handling the job according to the job frame. All resources in clouds are dynamic and scalable. Cloud computing ensures sharing of resources and common infrastructure to offer services to users, so that operations meet applications needs. Resource's/device's location is unknown to network's end user. Users can also develop/manage cloud applications with the cloud making resources virtualization by maintaining/managing itself. Rest of this paper is organized as follows in Section 2 discusses about BEE Scout technique in cloud computing environment description, Section 3 discusses about the proposed algorithm. Sections 4 describe the simulation process of proposed model and finally discuss the conclusion \&future scope in Section 5.

\section{BEE SCOUT}

The colonies of Bee play major role in problem optimization for the enhancement of process. The colonies of Bee create the Scout of leaving and new enter bee scenario. The $75 \%$ Bee leave the scout and execute the new process for the forging process. The bee scout process are used in the balancing of load and job scheduling for cloud computing[17]. The process of scout generation during the load balancing used the feasible solution of load provided by the load balancer. The Bee scout can't not produce the feasible solution of given load during the processing of balancing of load, the process of scout production proceed the new overload situation in terms of diversity and infeasible solution for the formation of balancer. The diversity of load and acceptance of scout give a constraints function for the processing of load describe here

$$
v i j=\left\{\begin{array}{c}
L i j+\emptyset i j(L i j-O i j), \text { if } R j \leq T R \\
L i j, \text { otherwise }
\end{array}\right.
$$

Here Vij is the feasible contain of Bee Scout and Lij is load matrix, Oij is proceed balance of load $\mathrm{Rj}$ is total available resource for the processing of job and TR is total resource of dedicated structure of cloud environments

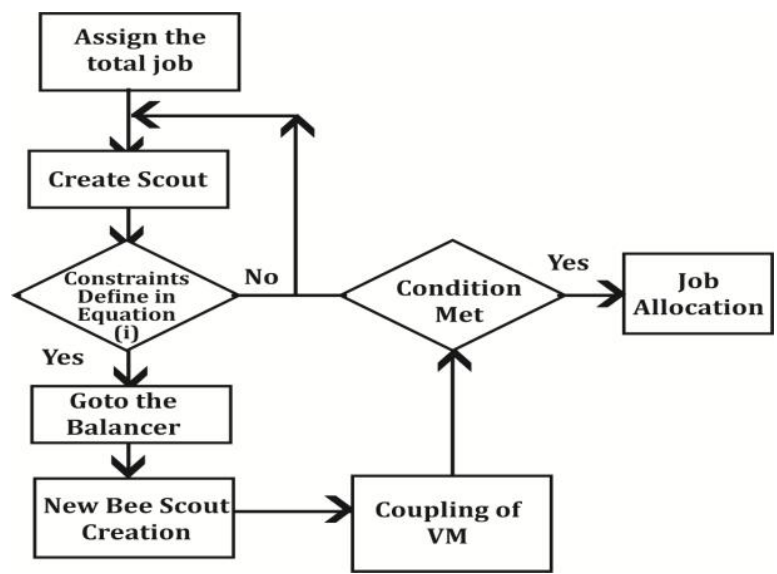

Figure 1 block diagram of Bee Scout and allocation of Job

\section{VIRTUAL MACHINE COUPLING (VMC)}

A method of coupling of virtual machines in a cloud computing environment, the cloud computing environment 
comprising a plurality of virtual machines ('VMs'), the VMs comprising modules of coupling computing machinery installed upon cloud computers disposed within a data center, the cloud computing environment further comprising a cloud operating system and a data center administration server operably coupled to the VMs, the method comprising. Deploying, by the cloud operating system, an instance of a $\mathrm{VM}$, including flagging the instance of a VM for autonomic scaling and executing a data processing workload on the instance of a VM[16].

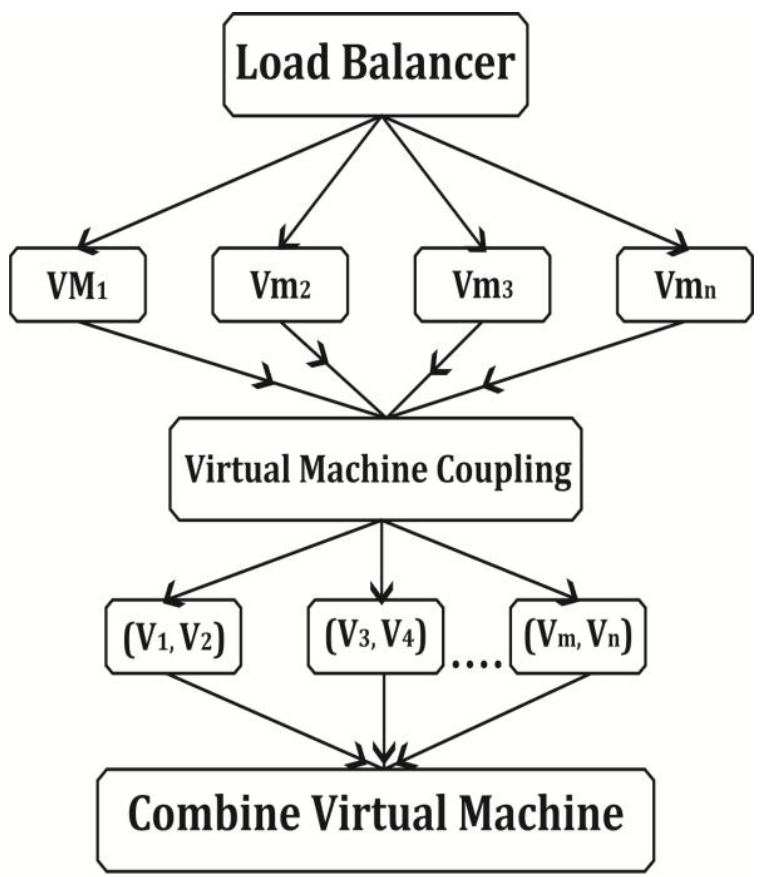

Figure 2: shows that process of coupling of virtual machine

\section{PROPOSED METHODOLOGY}

In this section discuss the load balancing model using honey Bee Scout process. The honey Bee scout precedes the dedicated load for the processing of job scheduling. The scheduling of job divide into two three section, one is ideal condition, another is under load condition and other is overload condition.

1. Find capacity loads of all VMs based on the three conditions define in Bee Scout job is under load.

If $S E<=T L$

Loader is balanced.

Exit

2 Create the decision node for allocation

If TDij> maximum capacity // if the total load of request matrix is maximum then the balancer is not working

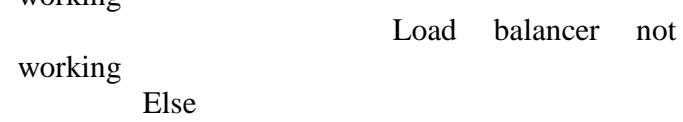

Call allocation process. // call function of Bee Scout for decision of request load.

1. Share all virtual machine capacity // coupling of virtual machine
2. Call decision factor: // call resource manager for mapping

Create node of VMs

$$
\text { supply of VMS = }
$$

Maximumm Capacity - $\frac{\text { Load }}{\text { Capacity }} / /$ estimate total capacity of couple virtual machine

Demand of each machine in node is

$$
\text { demand of } w i_{j}=\frac{\text { Load }}{\text { Capacity }}-
$$

Maximumm Capacity // SEij request matrix load per their request.

While $\mathrm{Ta} \neq \varphi$ and WIJ $\neq \varphi / /$ if total capacity and request matrix of load is null

For $s=1$ to \# (Ta) do //couple all virtual machine

Sort all VMs

For each task $\mathrm{T}$ in VMs find machine $V M_{d} \in T a$ such as

$$
T a \rightarrow V M_{d} \mid \min \left(\sum T\right) \in V M_{d} \text { and } \operatorname{Load}_{V M_{d}} \leq
$$
Capacity $_{V M_{d}} / /$ allocate request load to their resource.

$$
\text { If ( } \mathrm{T} \text { is allocated time) }
$$$$
T_{u} \rightarrow V M_{d} \mid \min \left(\sum T_{h}\right) \in V M_{d} / / \quad \text { single }
$$
machine allocation

$$
T_{o} \rightarrow V M_{d} \mid \min \left(\sum T_{h}+\sum T_{m}\right) \in V M_{d}
$$

coupled machine allocation

Finally, Tu and To is combined and transfer to data center for the execution of job.

\section{EXPERIMENTAL RESULT}

In this section the experimental process of proposed algorithm of load balancing. The proposed algorithm simulate in cloudsim simulator. The cloudsim simulator is java based software. The measuring of performance used average, minimum and maximum processing time of data centers.

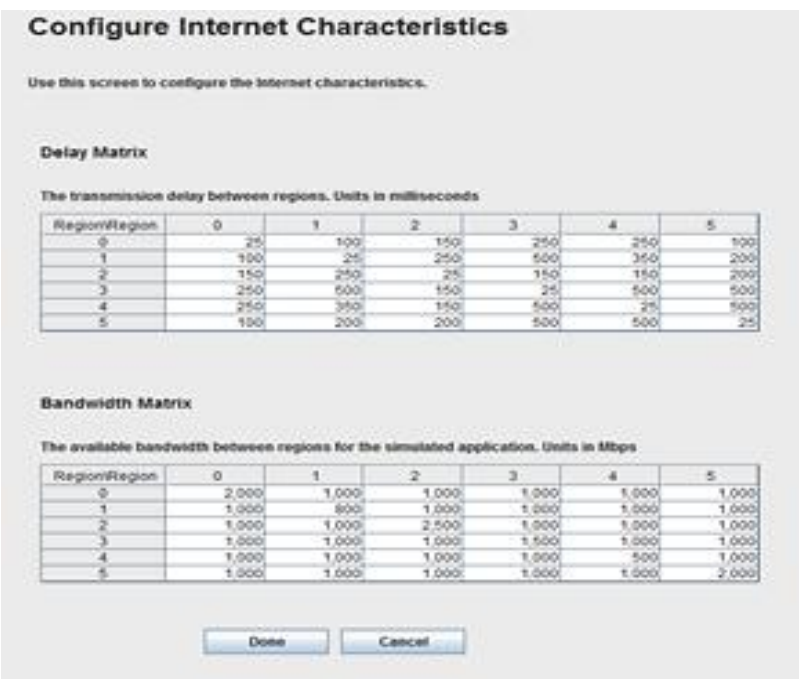

Figure 2: Shows the Internet Characteristics and it's configuration in Cloud Computing Environment. 


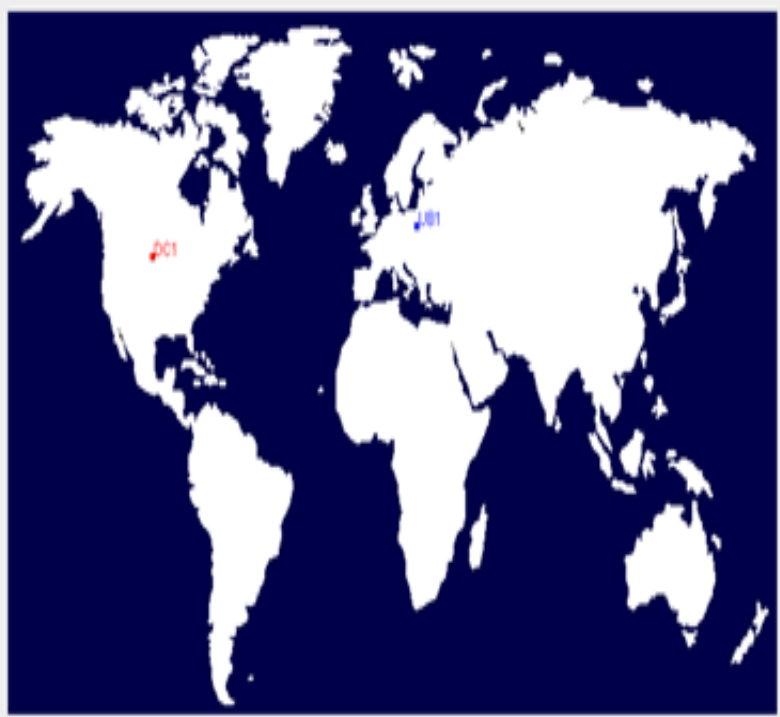

Figure 3: Shows the Data center and User Database Cloud Computing Environment

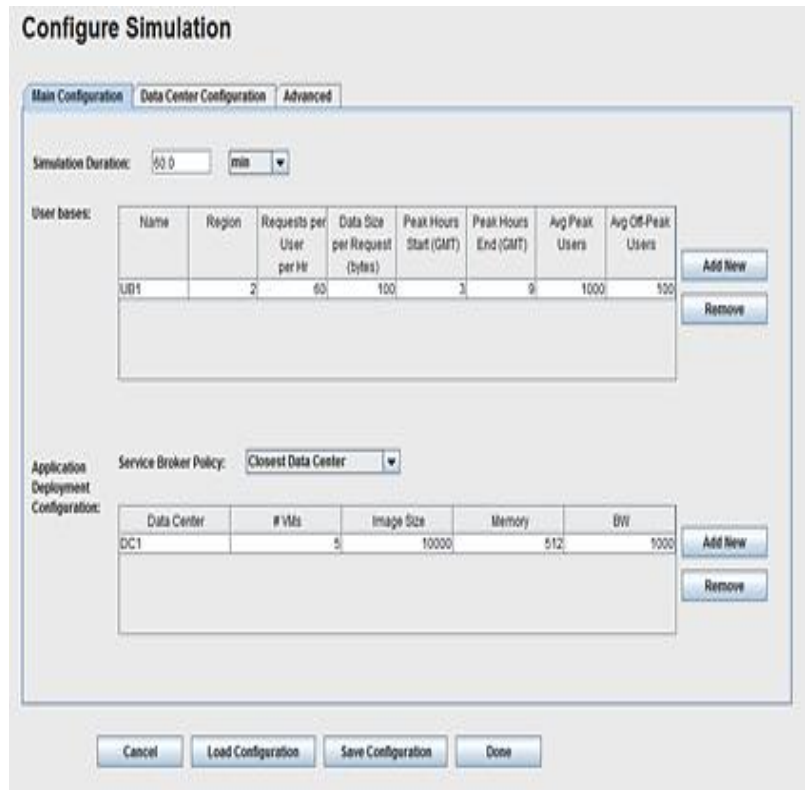

Figure 4: Shows the configuration Simulation of User Database and Data center in Cloud Computing Environment

Table 1: Shows the Overall Response Time analysis for CGT \& BEE Method

\begin{tabular}{|l|l|l|l|l|}
\hline Data Set & $\begin{array}{l}\text { Method } \\
\text { Name }\end{array}$ & Average & Minimum & Maximum \\
\hline $\begin{array}{c}\text { UB1,DC1 } \\
\& \\
\text { UB2,DC2 }\end{array}$ & CGT & 467.33 & 214.26 & 831.41 \\
\cline { 2 - 5 } & BEE & 423.45 & 195.26 & 780.23 \\
\hline
\end{tabular}

Table 2: Shows the DataCenter Processing Time analysis for CGT \& BEE Method

\begin{tabular}{|c|c|c|c|c|}
\hline Data Set & $\begin{array}{c}\text { Method } \\
\text { Name }\end{array}$ & Average & Minimum & Maximum \\
\hline $\begin{array}{c}\text { UB1,DC1 } \\
\& \\
\text { UB2,DC2 }\end{array}$ & CGT & 0.26 & 0.01 & 0.45 \\
\cline { 2 - 5 } & BEE & 0.18 & 0.01 & 0.32 \\
\hline
\end{tabular}

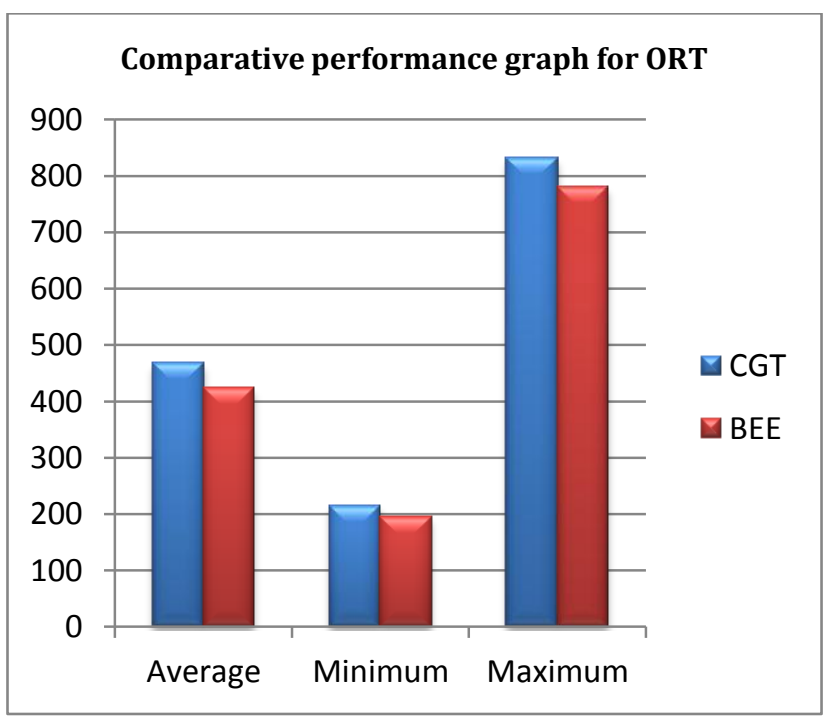

Figure 5: Shows that the comparative performance of ORT for UB1/DC1 \& UB2/DC2 using BEE Method which shows the better response time than methods such as CGT in terms of Average, Minimum and Maximum time in mili seconds

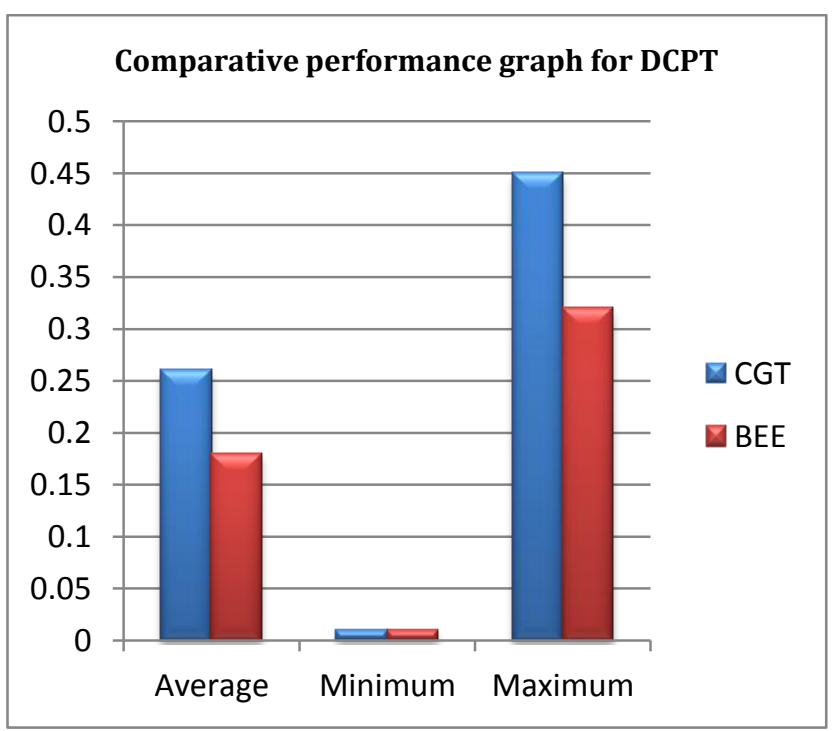

Figure 6: Shows that the comparative performance of DCPT for UB1/DC1 and UB2/DC2 using BEE Method which shows the better processing time than methods such as CGT in terms of Average, Minimum and Maximum time in mili seconds 


\section{CONCLUSION AND FUTURE WORK}

In this paper used Bee scout based algorithm for the selection of job and task for the resource allocation in public cloud computing. The BEE function gives the better performance instead of other CGT based algorithm such as game theory. Basically BEE algorithm facilities the all task in two sections one is job allocation process and other is job selection process. The job selection process is defined by the predefined constraints function. The predefined function selects the job on the basis of local scout functioning on the criteria of neighbors. For the evaluation of performance used cloud simulator software such is called cloud analyst. The cloud analysis software is bag of composition of cloud environment and load balancing policy. In scenario of policy design two services one is genetic algorithm policy and other is BEE based policy. The BEE based policy reduces the load effect approx. 22-25\% in compression of CGT. The modified load balancing policy is very efficient for public cloud computing.

\section{REFERENCES}

[1] Yasser Alharbi and Kun Yang "Optimizing jobs' completion time in cloud systems during Virtual Machine Placement", International Conference on Big Data and Smart City, 2016, Pp 1-6.

[2] Amir Nahir, Ariel Orda and Danny Raz "ReplicationBased Load Balancing”, IEEE, 2016, Pp 494-507.

[3] AlakaAnanth and K. Chandrasekaran "Cooperative Game Theoretic Approach for Job Scheduling in Cloud Computing", Computing and Network Communications, 2015, Pp 147-156.

[4] Matthew Malensek, SangmiPallickara, and ShrideepPallickara "Minerva: Proactive Disk Scheduling for QoS in Multitier, Multitenant Cloud Environments", IEEE, 2016, Pp 19-27.

[5] Nguyen KhacChien, Nguyen Hong Son and Ho DacLoc "Load Balancing Algorithm Based on Estimating Finish Time of Services in Cloud Computing", ICACT, 2016, Pp 228-233.

[6] Jing Tai Piao and Jun Yan "A Network-aware Virtual Machine Placement and Migration Approach in Cloud Computing”, IEEE, 2010, Pp 87-92.

[7] Kien Le, Jingru Zhang, JiandongMeng, Ricardo Bianchini, YogeshJaluria and Thu D. Nguyen "Reducing Electricity Cost Through Virtual Machine Placement in High Performance Computing Clouds", ACM, 2011, Pp $1-12$.

[8] KyongHoon Kim, Anton Beloglazov and RajkumarBuyya "Power-Aware Provisioning of Virtual Machines for Real-Time Cloud Services", John Wiley \& Sons, Ltd., 2011, Pp 1-19.

[9] Saurabh Kumar Garg, Adel NadjaranToosi, Srinivasa K.
Gopalaiyengar and RajkumarBuyya "SLA-based virtual machine management for heterogeneous workloads in a cloud datacenter", Journal of Network and Computer Applications, 2014, Pp 108-119.

[10] George Kousiouris, TommasoCucinotta and Theodora Varvarigou "The effects of scheduling, workload type and consolidation scenarios on virtual machine performance and their prediction through optimized artificial neural networks", The Journal of Systems and Software, 2011, Pp 1270-1291.

[11] DeepalJayasinghe, CaltonPu, Tamar Eilam, MalgorzataSteinder, Ian Whalley and Ed Snible "Improving Performance and Availability of Services Hosted on IaaS Clouds with Structural Constraint-aware Virtual Machine Placement”, IEEE, 2011, Pp 72-79.

[12] SriramKailasam, Nathan Gnanasambandam, JanakiramDharanipragada and Naveen Sharma "Optimizing Service Level Agreements for Autonomic Cloud Bursting Schedulers", Parallel Processing Workshops, 2010, Pp 285-294.

[13] ZeratulIzzahMohdYusoh and Maolin Tang "Composite SaaS Placement and Resource Optimization in Cloud Computing using Evolutionary Algorithms", IEEE, 2012, Pp 590-597.

[14] Abhishek Gupta, Laxmikant V. Kale, DejanMilojicic, Paolo Faraboschi and Susanne M. Balle "HPC-Aware VM Placement in Infrastructure Clouds”, IEEE, 2013, Pp 11-20.

[15] YueGao, Yanzhi Wang, Sandeep K. Gupta and MassoudPedram "An Energy and Deadline Aware Resource Provisioning, Scheduling and Optimization Framework for Cloud Systems", IEEE, 2013, Pp 1-10.

[16] Anton Beloglazov and RajkumarBuyya "Optimal Online Deterministic Algorithms and Adaptive Heuristics for Energy and Performance Efficient Dynamic Consolidation of Virtual Machines in Cloud Data Centers", John Wiley \& Sons, Ltd., 2011, Pp 1-24.

[17] Obaid Bin Hassan, A Sarfaraz Ahmad "Optimum Load Balancing of Cloudlets Using Honey Bee Behavior Load Balancing Algorithm" International Journal of Advance Research in Computer Science and Management Studies, 2015. Pp 334-338.

[18] SalimBitam "Bees Life Algorithm for Job Scheduling in Cloud Computing", ICCIT, 2012. Pp 186-191.

[19] Mohamed Firdhous, Osman Ghazali, Suhaidi Hassan, Nor ZiadahHarun, AziziAbas "honey bee based trust management system for cloud computing" Proceedings of the 3rd International Conference on Computing and Informatics, ICOCI, 2011.Pp 126-131. 